\title{
Indication of linkage and genetic heterogeneity for asthma and atopy on chromosomes $8 p$ and $12 q$ in 107 French EGEA families
}

\author{
M-H Dizier ${ }^{*, 1}, \mathrm{H}$ Quesneville ${ }^{2}$, C Besse-Schmittler ${ }^{3}$, M Guilloud-Bataille ${ }^{1}$, \\ $\mathrm{H}_{\text {Selinger-Leneman }}{ }^{1}$, F Clerget-Darpoux ${ }^{1}$ and F Demenais ${ }^{3}$
}

\begin{abstract}
${ }^{1}$ INSERM U535, Villejuif, France; ${ }^{2}$ Laboratoire dynamique du génome et évolution, Institut Jacques Monod, Paris, France; ${ }^{3}$ INSERM-EMI 00 06, Evry, France
\end{abstract}

Using the sample of 107 families with at least two asthmatic siblings, as part of the EGEA study, we have investigated linkage to asthma (or atopy) and genetic heterogeneity according to the presence/absence of atopy (or asthma) using two approaches: (1) the triangle test statistic (TTS), which considers the identical by descent (IBD) distribution among affected sib-pairs discordant for another associated phenotype (eg asthmatic sib-pairs discordant for atopy) and (2) the predivided sample test (PST), which compares the IBD distribution of marker alleles between affected sib-pairs concordant and discordant for the associated phenotype. Two regions, $8 p$ and $12 q$, already reported to be linked to both asthma and atopy, were examined here. A total of $\mathbf{2 0}$ asthmatic sib-pairs discordant for atopy and $\mathbf{2 4}$ atopic pairs discordant for asthma were analyzed by both TTS and PST methods and 83 pairs with atopic asthma by PST. Some evidence for linkage was observed for two markers in the 8p23.3-p23.2 region; D8S504 for asthma with genetic heterogeneity according to the presence/absence of atopy and D8S503 for atopy with genetic heterogeneity according to the presence/absence of asthma. In the 12q14.2-q21.33 region, there was also some evidence of linkage to two markers, D12S83 and D12S95, for atopy and asthma, respectively, with genetic heterogeneity according to the presence/absence of the associated trait. Provided the small distance between the two markers on either $8 p(16 \mathrm{cM})$ or $12 q(21 \mathrm{cM})$, it is unclear whether one or two genetic factors are involved in either region.

European Journal of Human Genetics (2003) 11, 590-596. doi:10.1038/sj.ejhg.5201014

Keywords: asthma; atopy; linkage; sib-pairs; genetic heterogeneity

Introduction

Asthma is a complex disorder that is likely to depend on numerous genetic and environmental factors. This disorder is also a heterogeneous disease which presents a large clinical spectrum. The pattern of asthma varies according to age and gender. We have already investigated genetic heterogeneity of asthma according to age at onset of

*Correspondence: Dr M-H Dizier, Hôpital Paul Brousse, Bâtiment Leriche, B.P. 1000, 94817 Villejuif Cedex, France. Tel: +33 1455953 91; Fax: +331 455953 31; E-mail : dizier@vjf.inserm.fr

Received 11 October 2002; revised 12 March 2003; accepted 18 March 2003 asthma that led to detect the $7 \mathrm{q}$ region involved in asthma with genotype relative risks differing according to age at the onset of disease. ${ }^{1}$ Asthma shows also phenotypical heterogeneity regarding its association with atopy (intrinsic/extrinsic asthma). Similarly, atopy may be associated or not with asthma. Such heterogeneity may have a genetic basis. It has been recently pointed out that in last decades, proportion of asthma cases attributable to atopy may have been overestimated, ${ }^{2}$ and consequently phenotypical heterogeneity between intrinsic and extrinsic asthma has been neglected in research on etiological mechanisms and risk factors for asthma. Most genomewide searches and candidate gene studies, which reported many regions linked to 
asthma and/or atopy, ${ }^{3}$ were conducted for asthma and/or atopy separately and did not take into account their association. From our knowledge, we were the only group to take into account association between asthma and atopy, and reported linkage of $8 \mathrm{p}$ region to asthma and atopy with genetic heterogeneity in CSGA family data (as part of Genetic Analysis Workshop GAW12). ${ }^{4}$

Another region of interest, the $12 \mathrm{q}$ region, has been consistently reported to be linked to atopy and/or asthma $^{5-10}$ by genomewide scans and candidate gene studies. ${ }^{11-15}$ This region indeed contains numerous candidate genes, such as interferon-gamma (IFNG), mast cell growth factor $(M G F)$, insulin-like growth factor-1 (IGF1), leukotriene A4 hydrolase $(L T A 4 H)$, transcription factors (NFYB and STAT6) and neuronal nitric oxide synthase gene (NOS1).

Using the sample of 107 French families selected through two asthmatic siblings as part of the EGEA study, a first genome screen for asthma and related phenotypes reported linkage of $12 \mathrm{q}$ to eosinophil counts (a phenotype associated with asthma and atopy) but not to asthma nor to atopy. ${ }^{8}$ No linkage of asthma, atopy or any associated phenotype to $8 \mathrm{p}$ was found. The goal of this paper was to investigate in these data, linkage of $8 p$ and $12 q$ regions to either asthma or atopy, while accounting for the presence/ absence of the associated trait (atopy or asthma). For this purpose, we used two different approaches. One of them is the triangle test statistic (TTS) ${ }^{16}$ developed to detect both linkage and intra-sib-pair genetic heterogeneity, that is, the sibs differing for a factor on which the genotype relative risks of the linked gene depend. This test has been applied to asthmatic sib-pairs discordant for atopy and to atopic sib-pairs discordant for asthma. The other approach is the predivided sample test (PST), proposed to detect both linkage and genetic heterogeneity, comparing the identity by descent (IBD) distribution of marker alleles between affected sib-pairs discordant and concordant for the associated trait.

\section{Material}

\section{Family data}

As part of the EGEA study, ${ }^{17,18}$ a sample of 119 families with at least two asthmatic siblings and at most one asthmatic parent was collected for a genomewide search of asthma and related phenotypes. Criteria met by asthmatic siblings of our 119 families were described elsewhere. ${ }^{18}$ After excluding families with insufficient DNA available or showing non-Mendelian transmission, the analyzed sample consisted of 107 families with a total of 493 individuals. In the 279 genotyped siblings of the 107 families, 59\% of siblings were male subjects and their mean age was $14.5 \pm 7.6(\mathrm{SD})$ years.

The protocol was approved by the ethical committee and subjects signed informed consent forms.

\section{Clinical evaluation}

Information on respiratory and allergic symptoms, medical history and environmental factors was recorded on a standardized questionnaire. The following tests were performed:

- skin-prick tests (SPTs) to 11 allergens, that is, cat, Dermatophagoides pteronyssinus, Cladosporium herbarum, Alternaria tenuis, timothy grass, olive, birch, Parieteria judaica, ragweed, Aspergillus and Blatella germanica;

- basal spirometry and methacholine bronchial challenge test.

\section{Genotyping}

We considered three markers on chromosome 8p (D8S504, D8S503 and D8S552) and eight markers on chromosome 12q (D12S83, D12S43, D12S92, D12S95, D12S338, D12S366, D12S342 and D12S97). All these markers were genotyped for our previous genome scan by using the methodology previously described. ${ }^{8}$ The region covered by the markers of chromosome $8 \mathrm{p}$ ranged between 0 and $26 \mathrm{cM}$ from pter, spanning the region $(18 \mathrm{cM}$ apart from pter) that was previously detected for genetic heterogeneity of asthma and atopy. ${ }^{4}$ The region covered by the $12 \mathrm{q}$ markers spanned approximately $106 \mathrm{cM}$. This region was chosen since it included potential linkages to asthma and associated phenotypes by previous genome scans.

\section{Phenotypes analyzed}

In our sample of families selected for the genomewide search, asthma was defined as follows: a positive response to at least one of the two questions ((1) Have you ever had attacks of breathlessness at rest with wheezing? (2) Have you ever had an asthma attack?) associated with either the presence of bronchial hyper-responsiveness (defined as a fall in baseline $\mathrm{FEV}_{1}$, the forced expiratory volume at $1 \mathrm{~s}$, $\geqslant 20 \%$ at $\leqslant 4 \mathrm{mg} / \mathrm{ml}$ methacholine or $\geqslant 15 \%$ increase of baseline $\mathrm{FEV}_{1}$ after bronchodilator use), and hospitalization for asthma in life or asthma therapy. The presence of atopy was detected by the SPT response (a positive response to at least one allergen corresponding to a difference of weal diameter $\geqslant 3 \mathrm{~mm}$ with the negative control). In the 279 genotyped siblings of the 107 families, $83.7 \%$ were asthmatic and $80.5 \%$ were atopic. The distribution of all independent sib-pairs according to their status, concordant or discordant for asthma and for atopy, is given in Table 1.

\section{Analysis \\ Linkage analysis by IBD sharing}

The principle of sib-pair linkage analysis is to assess the sharing of marker alleles IBD among affected sib-pairs and to conclude in favor of linkage if the observed IBD distribution differs from that expected under the null hypothesis of independent segregation of the disease and 
Table 1 Distribution of sib-pairs according to their status for asthma and atopy

\begin{tabular}{lrrr}
\hline & \multicolumn{3}{c}{ Atopy } \\
Phenotypes of the two sibs & $(+,+)$ & $(+,-)$ & Total \\
\hline Asthma & & & \\
$(+,+)$ & 83 & 20 & 103 \\
$(+,-)$ & 24 & 22 & 46 \\
Total & 107 & 42 & 149 \\
\hline
\end{tabular}

+: Affected, -: Unaffected.

markers. This comparison can be made with the MLS statistic, ${ }^{19}$ based on a maximum likelihood method that estimates the proportions of sib-pairs with 2, 1 or 0 marker allele(s) IBD (respectively $z 2, z 1$ and $z 0$ ), given the observed marker genotypes. It has been shown ${ }^{20}$ that, if a susceptibility gene is linked to the marker, the proportions $z 2, z 1$ and $z 0$ among affected sib-pairs are constrained within a triangle by $2 z 0 \geqslant z 1 \geqslant 0.5$, referred as the triangle constraints. $^{21}$ However, Dizier et $a l^{16}$ showed that if the phenotype of each member of a sib-pair is determined by different genetic models, that is, corresponding to different genotype relative risks (ratios of genotype-specific penetrances to the penetrance of at-risk homozygotes), then the triangle constraints may not be valid. This situation can occur when the two sibs differ for a factor that modifies the genotype relative risks for the disease. This factor may be specific to the phenotype itself, for example, severe $v s$ mild form of the disease, or may correspond to the presence/ absence of an associated trait or an environmental factor.

\section{TTS method}

The Triangle Test Statistic, ${ }^{16}$ called TTS, has been introduced to test whether the triangle constraints in marker IBD distribution among affected sib-pairs are fulfilled. This test is defined as:

$$
\mathrm{TTS}=\log _{10}\left(L\left(Z_{\mathrm{u}}\right) / L\left(Z_{\mathrm{c}}\right)\right)
$$

where $L\left(Z_{\mathrm{c}}\right)$ is the likelihood of the IBD vector estimated with the triangle constraints and $L\left(Z_{\mathrm{u}}\right)$ is the likelihood without these constraints. The null hypothesis $\mathrm{HO}$ is composite: absence of linkage or linkage with genetic homogeneity (ie relative penetrances are the same for the phenotypes of both members of the sib-pair). Rejection of HO means linkage with genetic heterogeneity within sibpairs, that is, the sibs differ for a factor on which the relative penetrances depend. The TTS allows detection of linkage only in the presence of heterogneity.

As Holmans ${ }^{21}$ did for the MLS distribution, we used the method of Self and Liang $^{22}$ to calculate the asymptotic distribution of $2 \operatorname{Ln}(10)$.TTS (i.e. $2 \operatorname{Ln}\left(L\left(Z_{\mathrm{u}}\right) / L\left(Z_{\mathrm{c}}\right)\right)$ ) as a mixture of $\chi_{1 \mathrm{df}}^{2}$ and $\chi_{2 \mathrm{df}}^{2}$. Derivation of TTS distribution and table of thresholds for various sizes are described in
Dizier et al. ${ }^{1,16}$ We checked that critical $P$-values for given thresholds estimated from the asymptotic distribution were close to the empirical $P$-values calculated by simulations for small sample sizes of 20-24 discordant sib-pairs.

\section{The predivided sample test}

Let us consider two subphenotypes, $\mathrm{A}$ and $\mathrm{A}^{\prime}$, which represent the affected status with either absence/presence of a given factor (here asthma with or without atopy and atopy with and without asthma). Under the null hypothesis of genetic homogeneity (ie presence of the factor does not modify the relative penetrances), the $Z_{\mathrm{u}}$ vectors estimated, without the triangle constraints, among pairs concordant for the phenotype (AA or $\mathrm{A}^{\prime} \mathrm{A}^{\prime}$ ) and among discordant pairs (one sib is $A$, the other $A^{\prime}$ ) are expected to be equal. Therefore, the null hypothesis can be tested by $-2\left[\operatorname{Ln}\left(L\left(z_{\mathrm{u}} 1\right) \cdot L\left(z_{\mathrm{u}} 2\right) \cdot L\left(z_{\mathrm{u}} 3\right) / L\left(z_{\mathrm{u}}\right)\right)\right]$, which follows a $\chi^{2}$ distribution with $4 \mathrm{df},{ }^{23}$ where $L\left(z_{\mathrm{u}} 1\right), L\left(z_{\mathrm{u}} 2\right), L\left(z_{\mathrm{u}} 3\right)$ and $L\left(z_{\mathrm{u}}\right)$ are the likelihoods of the parameter vector, $Z_{\mathrm{u}}$, estimated, respectively, in the concordant sib-pairs for phenotype A, the concordant pairs for $\mathrm{A}^{\prime}$, the discordant pairs and the whole sample. The PST allows detection of both linkage and heterogeneity and, as the TTS, does not detect linkage in the absence of heterogeneity.

\section{Strategy of analysis}

In order to improve the power to detect genetic heterogeneity of asthma because of the presence/absence of atopy, we applied the TTS to asthmatic pairs discordant for atopy instead of considering all asthmatic sib-pairs. The PST was applied to asthmatic pairs concordant and discordant for atopy. However, since the number of concordant sib-pairs with the two sibs being both asthmatic and nonatopic was too small, this category was not considered, and thus the number of df of the test was 2 instead of 4 .

The same strategy was applied using atopy as the main phenotype and asthma as the stratifying one. The number of concordant sib-pairs with the two sibs being both atopic and non asthmatic being too small, this category was also ignored for PST.

These tests were applied using all possible independent pairs in sibships (ie $(n-1)$ pairs per sibships with $n$ affected sibs) and taking into account the information provided by the entire set of chromosome markers. The Bonferroni correction was applied to account for multiple testing (11 markers, three on $8 p$ and eight on $12 q)$. Note that this correction ignores linkage between markers and for this reason is conservative, overestimating the type-one error.

\section{Results}

Results for the $8 \mathrm{p}$ region are given in Table 2, which presents the markers with significant genetic heterogeneity (at 5\% level after Bonferroni correction) for at least one of 
the tests (TTS or PST). Among asthmatic sib-pairs discordant for atopy, a significant TTS score was obtained at D8S504 locus, with a TTS value of $2.3(P=0.003$, $P=0.03$ after correction). Genetic heterogeneity because of the presence/absence of atopy at this locus was confirmed by the PST that compared the IBD distribution between asthmatic pairs concordant and discordant for atopy ( $P=0.004, P=0.04$ after correction). Genetic heterogeneity at another locus D8S503, $16 \mathrm{cM}$ apart from the marker D8S504, was detected by the PST applied to atopic pairs concordant and discordant for asthma $(P=0.003$, $P=0.033$ after correction). Such heterogeneity was also suggested by the TTS test in atopic sib-pairs discordant for asthma $(P=0.03, P=0.33$ after correction), this result being no longer significant after correction for multiple testing.

The IBD distributions in the different types of sib-pairs for the two $8 \mathrm{p}$ markers detected by the TTS and/or the PST and adjacent markers are given in Table 3. Considering the IBD distribution at D8S504 in asthmatic sib-pairs discordant for atopy, there was a large departure from the triangle constraint $2 z 0 \leqslant z 1$ (with $z 0=0.57$ and $z 1=0.43$ ) explaining the rejection of genetic homogeneity by the TTS and the PST. There was also a slight excess of pairs sharing two

Table 2 Results of the TTS and the PST for markers of the $8 p$ region with a TTS $>1.7$

\begin{tabular}{|c|c|c|c|c|}
\hline Markers & TTS & P-value* & PST & P-value* \\
\hline \multicolumn{5}{|c|}{$\begin{array}{l}\text { Sample of asthmatic sib-pairs } \\
\text { discordant for atopy }\end{array}$} \\
\hline D8S504 & 2.3 & $0.003(0.03)$ & 11 & $0.004(0.04)$ \\
\hline \multicolumn{5}{|c|}{$\begin{array}{l}\text { Sample of atopic sib-pairs } \\
\text { discordant for asthma }\end{array}$} \\
\hline D8S503 & 1.8 & $0.03 \quad(0.33)$ & 11.8 & $0.003(0.03)$ \\
\hline
\end{tabular}

*P-values after the Bonferroni correction are given in parentheses. marker alleles IBD in asthmatic sib-pairs concordant for atopy with $z 2$ estimated at 0.29 compared to the expected value of 0.25 under no linkage. For D8S503 marker, in atopic sib-pairs discordant for asthma, there was departure from the constraint $z 1 \leqslant 0.5$ with $z 1$ being equal to 0.72 and an excess of pairs with $\mathrm{IBD}=2$ in atopic sib-pairs concordant for asthma $(z 2=0.35)$.

Results for the $12 \mathrm{q}$ region are given in Table 4 , with markers showing significant genetic heterogeneity (at 5\% level after Bonferroni correction) for at least one of the tests (TTS or PST). A significant TTS score was obtained in the asthmatic sib-pairs discordant for atopy at the locus D12S83, with a TTS value of $2.3(P=0.003, P=0.03$ after correction). Genetic heterogeneity of asthma owing to the presence/absence of atopy was suggested by the PST $(P=0.015, P=0.16$ after correction), which was no longer significant after correction of multiple testing. The TTS test in atopic sib-pairs discordant for asthma led to detect another locus linked to D12S95, 21 cM apart from the marker D12S83, with a TTS score of $2.2(P=0.003$, $P=0.033$ after correction). Such heterogeneity was also suggested by the PST $(P=0.015, P=0.16$ after correction), although, as previously, no longer significant after correction.

Table 4 Results of the TTS and the PST for markers of the $12 q$ region with a TTS $>1.7$

\begin{tabular}{|c|c|c|c|c|}
\hline Markers & TTS & P-value & PST & P-value* \\
\hline \multicolumn{5}{|c|}{$\begin{array}{l}\text { Sample of asthmatic sib-pairs } \\
\text { discordant for atopy }\end{array}$} \\
\hline D12S83 & $2.3^{\prime}$ & $0.003(0.03)$ & 8.3 & $0.015(0.16)$ \\
\hline \multicolumn{5}{|c|}{$\begin{array}{l}\text { Sample of atopic sib-pairs } \\
\text { discordant for asthma }\end{array}$} \\
\hline D12S95 & 2.2 & $0.003(0.03)$ & 8.3 & $0.015(0.16)$ \\
\hline
\end{tabular}

* $P$-values after the Bonferroni correction are given in parentheses.

Table 3 IBD distribution in sib-pairs at the markers D8S504 and D8S503 and adjacent markers

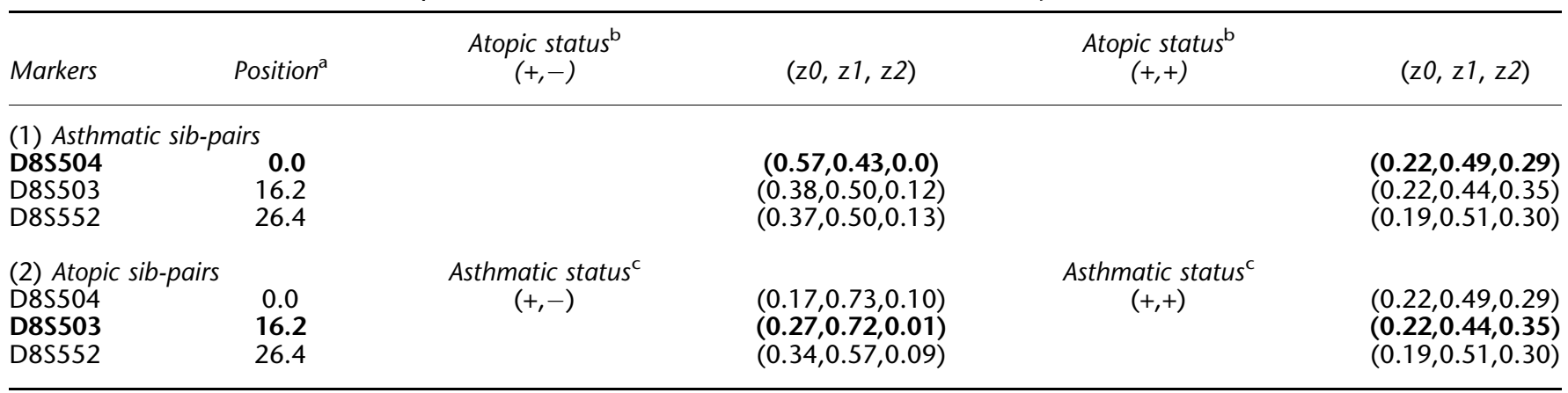

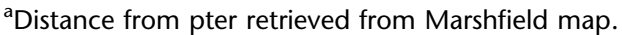

Markers showing genetic heterogeneity are presented in bold.

${ }^{\mathrm{b}}$ Atopic status of the two asthmatic sibs.

${ }^{\mathrm{c}}$ Asthmatic status of the two atopic sibs.

+ : Affected, -: Unaffected. 
Table 5 IBD distribution in sib-pairs at the markers D12S83 and D12S95 and adjacent markers

\begin{tabular}{|c|c|c|c|c|c|}
\hline Markers & Position $^{\mathrm{a}}$ & $\begin{array}{l}\text { Atopic status }^{\mathrm{b}} \\
(+,-)\end{array}$ & $(z 0, z 1, z 2)$ & $\begin{array}{l}\text { Atopic status }^{\mathrm{b}} \\
(+,+)\end{array}$ & $(z 0, z 1, z 2)$ \\
\hline $\begin{array}{l}\text { (1) Asthmatic sib-po } \\
\text { D12S87 } \\
\text { D12S83 } \\
\text { D12S43 }\end{array}$ & $\begin{array}{l}52 \\
75.2 \\
80.5\end{array}$ & & $\begin{array}{c}(0.32,0.47,0.21) \\
(0.21,0.79,0.0) \\
(0.32,0.52,0.16)\end{array}$ & & $\begin{array}{l}(0.16,0.62,0.22) \\
(0.21,0.56,0.23) \\
(0.22,0.56,0.22)\end{array}$ \\
\hline $\begin{array}{l}\text { (2) Atopic sib-pairs } \\
\text { D12S92 } \\
\text { D12S95 } \\
\text { D12S338 }\end{array}$ & $\begin{array}{r}83.2 \\
96.1 \\
111.9\end{array}$ & $\begin{array}{c}\text { Asthmatic status }{ }^{\mathrm{c}} \\
(+,-)\end{array}$ & $\begin{array}{l}(0.10,0.74,0.16) \\
(0.08,0.82,0.09) \\
(0.31,0.46,0.23)\end{array}$ & $\begin{array}{c}\text { Asthmatic status }{ }^{\mathrm{c}} \\
(+,+)\end{array}$ & $\begin{array}{l}(0.22,0.55,0.23) \\
(0.26,0.48,0.26) \\
(0.25,0.44,0.31)\end{array}$ \\
\hline
\end{tabular}

See Table 3.

Considering the IBD distributions at D12S83 marker and adjacent markers in asthmatic sib-pairs discordant for atopy (Table 5), there was a large departure from the constraint $z 1 \leqslant 0.5(z 1=0.79)$, which explained the rejection of genetic homogeneity by the TTS. However, there was no distortion from the expected IBD distribution under no linkage $(0.25,0.5,0.25)$, among asthmatic sib-pairs concordant for atopy. Similar findings were observed at D12S95 in atopic sib-pairs, with a proportion $z 1(=0.82)$ much greater than 0.5 in atopic pairs discordant for asthma but no distortion from random IBD distribution in atopic sib-pairs concordant for asthma.

\section{Discussion}

Using two tests, the TTS and the PST, which can detect both linkage and genetic heterogeneity, the two regions 8p23.3-p23.2 and 12q14.2-q21.33 were each found linked to asthma while taking into account presence/ absence of atopy and to atopy while taking into account presence/absence of asthma in our sample of 107 EGEA families. Our previous screen $^{8}$ did not indicate any evidence for linkage of these regions to asthma and atopy using a model-free linkage method applied separately to each of the two phenotypes. Linkage to chromosome $12 \mathrm{q}$ was suggested for eosinophil counts but in a region, respectively, 37 and $58 \mathrm{~cm}$ apart from the two markers detected here. Accounting for possible heterogeneity when it exists permits to increase power to detect linkage. ${ }^{16}$ As shown in our previous work, ${ }^{16}$ some genetic models of heterogeneity, where a factor affects linkage to a disease, can lead to the following distributions: an excess of pairs $\mathrm{IBD}=1$ and/or of pairs $\mathrm{IBD}=0$ with no excess of pairs $\mathrm{IBD}=2$ (even sometimes a proportion of pairs IBD $=2$ close to 0 ) in affected pairs discordant for the factor and no or only small excess of pairs $\mathrm{IBD}=2$ in affected sib-pairs concordant with the factor. These types of distribution correspond to those observed for the two regions investigated here, the $8 p$ and $12 q$ regions. In such cases and with these types of affected sib-pairs, no linkage could be detected by methods using only excess of allele sharing, while the TTS or PST could detect both linkage and heterogeneity.

The $8 \mathrm{p}$ region has been reported to be linked to atopy by one genome scan in pedigrees from the United Kingdom, ${ }^{24}$ but was not found in any other published genome scans using classical linkage analysis. However, it was also detected $^{4}$ by using the same approach as the one applied here, that is, accounting for phenotypical heterogeneity of asthma and atopy with the TTS and the PST, in a sample of Caucasian North American families of the CSGA study ${ }^{5}$ (data available for GAW12). This study allowed to conclude to a genetic factor in the $8 \mathrm{p}$ region involved in atopy with genetic heterogeneity according to the presence/absence of asthma. This result is replicated by the present study using the French EGEA data, with some evidence for genetic heterogeneity at two markers within this region. The first marker D8S504 was detected for linkage to asthma with genetic heterogeneity according to the presence/absence of atopy, both by the TTS in asthmatic sib-pairs discordant for atopy and by the PST, which compared asthmatic pairs concordant and discordant for atopy. The second marker D8S503, which is $16 \mathrm{~cm}$ apart from D8S504, was detected for linkage to atopy with genetic heterogeneity according to the presence/absence of asthma with the PST test. It was also suggested by the TTS, although the result was no longer significant after correction for multiple tests. We cannot exclude the possibility that there are two genetic factors in this region. However, given the length of regions detected by linkage analyses of complex traits, it is more likely that there is only one genetic factor in a $16 \mathrm{cM}$ region.

Similar findings were observed for markers on chromosome $12 \mathrm{q}, \mathrm{D} 12 \mathrm{~S} 83$ detected for atopy with genetic heterogeneity according to the presence/absence of asthma and D12S95 detected for asthma with genetic heterogeneity according to the presence/absence of atopy. However, it is not obvious to conclude whether one or two genetic 
factors are involved, provided the two potential linkages, which are $21 \mathrm{~cm}$ apart, are separated by two markers showing no genetic heterogeneity. Moreover, given the numerous candidate genes located on chromosome 12q, several of them may be involved. The first detected marker D12S83 is $5 \mathrm{cM}$ apart from $I F N G$, while the second marker D12S95 is close to SCF and NFYB (1 cM apart). Note that most previouly published linkages to $12 \mathrm{q}$ for asthma and atopy $^{5-7,11-13,15}$ were located within the region flanked by D12S83 and D12S95 markers. A study ${ }^{14}$ found linkage for asthma to $12 \mathrm{q}$ in another region, the same that was detected by our previous screen for eosinophils ${ }^{8}$ (37 and $54 \mathrm{~cm}$ apart from D12S83 and D12S95, respectively), this region including the candidate gene NOS1. Also, two genome scans ${ }^{9,10}$ have recently detected linkage to $12 \mathrm{q}$ for asthma and $\operatorname{IgE}$ at markers located between these two regions.

Rejection of homogeneity by the PST for the $12 \mathrm{q}$ markers was no longer significant after correction for multiple testing, while it remained significant by TTS. There was indeed no distortion from random distribution of the IBD distribution in concordant pairs for $12 \mathrm{q}$ markers. Such distortion exists for the markers of the $8 \mathrm{p}$ region and could explain in part the stronger rejection of homogeneity by the PST for the $8 p$ markers than for the $12 q$ markers. In contrast, for $8 \mathrm{p}$ markers, genetic heterogeneity was signicantly detected by the PST but not by the TTS (after correction for multiple tests) for one of the markers. These results suggest different underlying models for the genetic factors located within the two regions $8 \mathrm{p}$ and $12 \mathrm{q}$. Our results also stress the interest of using the two tests (TTS and PST) simultaneously.

Detection of linkage and genetic heterogeneity using the TTS was found at the markers D12S83 and D8S504, in asthmatic sib-pairs discordant for atopy. When considering all sib-pairs discordant for atopy (whichever be their asthmatic status), the TTS did not lead to a significant heterogeneity (results not shown). Similar findings were observed at loci D12S95 and D8S503 where there was no significant TTS results in all sib-pairs discordant for asthma (whatever be the atopic status), while there was a significant TTS result in the subset of atopic sib-pairs discordant for asthma. These results suggest that the linked genetic factors detected on $8 \mathrm{p}$ and $12 \mathrm{q}$ chromosomes are involved in both atopy and asthma. The strong but not complete association between the two traits may result from both common determinants and specific ones to each of the phenotypes. The present work may have been able to show potential evidence for some genetic factor(s) on chromosomes $8 \mathrm{p}$ and $12 \mathrm{q}$ which could partly explain the association between asthma and atopy.

\section{EGEA cooperative group}

Respiratory epidemiology: INSERM U472, Villejuif: I AnnesiMaesano, F Kauffmann (coordinatrice), MP Oryszczyn;
INSERM U408, Paris: M Korobaeff, F Neukirch. Genetics: INSERM EMI 00 06, Evry: F Demenais; INSERM U535, Kremlin-Bicêtre: MH Dizier; INSERM U393, Paris: J Feingold; CNG, Evry: M Lathrop.Clinical centers: Grenoble: I Pin, C Pison; Lyon: D Ecochard (deceased), F Gormand, Y Pacheco; Marseille: D Charpin, D Vervloet; Montpellier: J Bousquet; Paris Cochin: A Lockhart, R Matran (actuellement à Lille); Paris Necker: E Paty, P Scheinmann; ParisTrousseau: A Grimfeld.

Data managements: INSERM ex-U155, Paris: J Hochez; INSERM U472 : N Le Moual.

\section{Acknowledgements}

This work was supported by INSERM grant (IDS-4D006D). Kits for IgE and Phadiatop ${ }^{\circledR}$ determinations were kindly provided by Pharmacia.

\section{References}

1 Dizier MH, Besse-Schmittler C, Guilloud-Bataille $\mathrm{M}$ et al: Asthma according to age at onset on chromosome $7 q$ in 107 French EGEA families. Eur J Hum Genet 2001; 9: 867-872.

2 Pearce N, Pekkanen J, Beasley R: How much asthma is really attributable to atopy? Thorax 1999; 54: 268-272.

3 Ober C, Moffatt M: Contributing factors to the pathobiology: the genetics of asthma. in Wenzel S (ed). The pathobiology of asthma. WB Saunders; Philadelphia, 2000, vol 21, pp 245-261.

4 Dizier $\mathrm{MH}$, Babron MC: Triangle test statistic in discordant sibpairs: test of genetic homogeneity of asthma and atopy in CSGA families. Genet Epidemiol 2001; 21: S192-S197.

5 The Collaborative Study on the Genetics of Asthma (CSGA): A genome-wide search for asthma susceptibility loci in ethnically diverse populations. Nat Genet 1997; 15: 389-392.

6 Ober C, Cox NJ, Abney M et al: Genome-wide search for asthma susceptibility loci in a founder population. Hum Mol Genet 1998; 7: 1393-1398.

7 Wjst M, Fisher G, Immervoll T et al: A genome-wide search for linkage to asthma. Genomics 1999; 58: 1-8.

8 Dizier MH, Besse-Schmittler C, Guilloud-Bataille M et al: Genome screen for asthma and related phenotypes in the French EGEA study. Am J Respir Crit Care Med 2000; 162: 1812-1818.

9 Xu J, Postma DS, Howard TD et al: Major genes regulating total serum immunoglobulin E levels in families with asthma. Am J Hum Genet 2000; 67: 1163-1173.

$10 \mathrm{Xu} \mathrm{J}$, Meyers DA, Ober C et al: Genomewide screen and identification of gene-gene interactions for asthmasusceptibility loci in three U.S. populations: Collaborative Study on the Genetics of Asthma. Am J Hum Genet 2001; 68: 1437-1446.

11 Barnes KC, Neely JD, Duffy DL et al: Linkage of asthma and total serum IgE concentration to markers on chromosome 12q: evidence from Afro-Caribbean and Caucasian populations. Genomics 1996; 37: 41-50.

12 Barnes KC, Freidhoff LR, Nickel R et al: Dense mapping of chromosome 12q13.12-q23.3 and linkage to asthma and atopy. J Allergy Clin Immunol 1999; 104: 485-491.

13 Nickel R, Wahn U, Hizawa $\mathrm{N}$ et al: Evidence for linkage of chromosome 12q15-q24.1 markers to high total serum IgE concentrations in children of the German multicenter allergy study. Genomics 1997; 46: 159-162.

14 Wilkinson J, Grimley S, Collins A et al: Linkage of asthma to markers on chromosome 12 in a sample of 240 families using quantitative phenotype scores. Genomics 1998; 53: 251-259.

15 Blumenthal MN, Rich SS, King R, Weber J and the Collaborative Study for the Genetics of Asthma (CSGA): Approaches and issues 
in defining asthma and associated phenotypes map to chromosome susceptibility areas in large Minnesota families. Clin Exp Allergy 1998; 28: 51-55.

16 Dizier MH, Quesneville H, Prum B, Selinger-Leneman H, ClergetDarpoux F: The triangle test statistic (TTS): a test of genetic homogeneity using the departure from the triangle constraints in IBD distribution among affected sib-pairs. Ann Hum Genet 2000; 64: 433-442.

17 Kauffmann F, Dizier MH, Pin I et al: Epidemiological study on the genetics and environment of asthma bronchial hyperresponsiveness and atopy (EGEA) - phenotype issues. Am J Respir Crit Care Med 1997; 156: S123-S129.

18 Kauffmann F, Dizier MH, Annesi-Maesano I et al: Etude épidémiologique des facteurs Génétiques et Environnementaux de l'Asthme, l'hyperréactivité bronchique et l'atopie (EGEA) Protocole et biais de sélection potentiels. Rev Epidémiol Santé Pub 2001; 49: 343-356.
19 Risch N: Linkage strategies for genetically complex traits III. The effect of Marker polymorphism on analysis of affected relative pairs. Am J Hum Genet 1990; 46: 242-253.

20 Suarez BK: The affected sib pair IBD distribution for HLA-linked disease susceptibility genes. Tissue Antigens 1978; 12: 87-93.

21 Holmans P: Asymptotic properties of affected sib-pair linkage analysis. Am J Hum Genet 1993; 52: 362-374.

22 Self SG, Liang KY: Asymptotic properties of maximum likelihood estimators and likelihood ratio tests under non-standard conditions. J Am Stat Assoc 1987; 82: 605-610.

23 Morton NE: The detection and estimation of linkage between the genes for elliptocytosis and the Rh blood type. Am J Hum Genet 1956; 8: 80-96.

24 Strauch K, Fimmers R, Kurz T, Deichmann KA, Wienker TF, Baur MP: Parametric and nonparametric multipoint linkage analysis with imprinting and two-locus-trait-models: application to mite sensitization. Am J Hum Genet 2000; 66: 1945-1957. 